\title{
Intubation Checklist for COVID-19 Patients: A Practical Tool for Bedside Practitioners
}

\author{
Alfred Papali, Anna O Ingram, Amy M Rosenberger, Frances R D’Arcy, Jaspal Singh, \\ Lisa Ahlberg, and Christopher D Russell
}

\begin{abstract}
BACKGROUND: The high frequency of aerosolizing procedures in the ICU, including endotracheal intubation, places clinical staff at elevated risk of contracting the 2019 coronavirus (COVID-19). Use of an intubation checklist can reduce exposure risk and thus acts as a potential safeguard. Specific, step-by-step guidance to perform safe endotracheal intubation in the setting of COVID-19 are limited. This article outlines the development and refinement of a COVID-19 intubation checklist and operational protocol for ICU staff at a single center in the United States. METHODS: A standard pre-intubation checklist was adapted and refined by consensus using a multidisciplinary and iterative process, then distributed to local staff for clinical use. Subsequent mock intubation training sessions were held using the new checklist to ensure proficiency. Planned debriefing sessions helped identify several previously unanticipated issues, allowing for further refinement of the intubation checklist and inclusion of all stakeholders. RESULTS: A COVID-19 intubation checklist helped optimize safety during a high-risk situation by minimizing aerosolization of secretions, the number of staff required in the room, the time spent in the room, and the frequency of donning/doffing personal protective equipment. CONCLUSIONS: We present a checklist for use during high-risk intubations of COVID-19 patients, which serves as a pragmatic bedside tool for clinicians. The process of checklist development may also serve as a model for facilities preparing their own pandemic protocols. Key words: intubation; COVID; pandemic; checklist; ICU; safety; protocol; quality improvement. [Respir Care 2021;66(1):138-143. (C) 2021 Daedalus Enterprises]
\end{abstract}

\section{Introduction}

The 2019 coronavirus (COVID-19) pandemic has forced a rapid, global re-assessment of hospital protocols to optimize protection of patients and health care workers alike. In the ICU, the high frequency of aerosolizing procedures, including endotracheal intubation, places clinical staff at

\footnotetext{
Drs Papali, Singh, and Russell, and Ms Ahlberg are affiliated with the Division of Pulmonary \& Critical Care Medicine, Atrium Health, Charlotte, North Carolina. Dr Papali is affiliated with the Division of Pulmonary, Critical Care and Sleep Medicine, University of North Carolina School of Medicine, Chapel Hill, North Carolina. Dr Singh is affiliated with the Levine Cancer Institute, Atrium Health, Charlotte, North Carolina. Ms Rosenberger, Ms Ingram, and Ms D'Arcy are affiliated with the Critical Care Nursing, Atrium Health, Charlotte, North Carolina.
}

Supplementary material related to this paper is available at http://www. rcjournal.com. particularly elevated risk of contracting hospital-acquired infection. ${ }^{1}$ Experience from prior pandemics suggests that following strict checklist protocols during high-risk situations can reduce health care workers' risk of exposure. ${ }^{2}$ Similarly, protocol deviations can increase risk. ${ }^{3}$ Outside of pandemic situations, protocolization in the form of checklists is universally accepted to improve the safety of surgery. ${ }^{4}$ In critical care, checklists improve the quality of advanced cardiac life support, ${ }^{5}$ adherence to sepsis best practices ${ }^{6}$ and incidence of bloodstream infections

\footnotetext{
Dr Singh has disclosed relationships with Medtronic, and Somnoware Sleep Solutions. All other authors have disclosed no conflicts of interest.

Correspondence: Alfred Papali MD, Atrium Health Pineville Hospital 10650 Park Road, Suite 300, Charlotte, NC 28210. E-mail: alfred.papali@atriumhealth.org.
}

DOI: $10.4187 /$ respcare. 08063 
associated with a central line. ${ }^{7}$ Preliminary studies regarding the benefits of intubation checklists have demonstrated mixed results, ${ }^{8,9}$ and the available literature for airway management specific to high-containment pathogens is either discordant with evolving best practices ${ }^{10}$ or lacks utility for use as a bedside tool. ${ }^{11-14}$

Intubation in a critically ill patient is a high-stress, timesensitive procedure that can result in significant patient harm if performed improperly, but there are several additional considerations for intubating a patient with COVID-19. The high risk of viral transmission requires proper donning of personal protective equipment (PPE), which can lead to delays, decreased number of staff at bedside, and strained communication between the bedside team and those assisting with equipment. An intubation checklist thus acts as a potential safeguard to lessen the risk of these concerns having a negative impact on patient care. Specific, step-by-step guidance for bedside clinicians to perform safe endotracheal intubation in the setting of highly infectious diseases, especially those transmitted by aerosolization of secretions, are limited. This article outlines the development and refinement of a COVID-19 intubation checklist and operational protocol for ICU staff at a single center in the United States. Our hope is that the checklist functions as a pragmatic bedside tool for practitioners and that the process of checklist development described herein serves as a model for facilities preparing their own pandemic protocols.

\section{Methods}

Atrium Health is a highly integrated, not-for-profit health care system in the Southeast United States with $>40$ hospitals, 900 care locations throughout the Carolinas and Georgia, and a robust tele-critical care program known as Virtual Critical Care. The ICU staff at Atrium Health Pineville, a tertiary care, community hospital near Charlotte, North Carolina, use a printed pre-intubation checklist, verbally completed just prior to intubation by a dedicated code nurse, for usual care (see the supplementary materials at http://www.rcjournal.com). As part of its COVID-19 pandemic response and prior to receiving its first patient with COVID, this intubation checklist was adjusted by a local physician and reviewed by intensivists at multiple sites within the Atrium Health system. The checklist was amended and finalized by consensus, then distributed to local nursing and respiratory therapy staff for clinical use. Institutional review board approval was not sought because the checklist was considered a necessary and urgent quality-improvement initiative specific to the COVID-19 pandemic.

To examine its application in a real-life scenario, the new intubation checklist was used first on a patient who did not have COVID-19 but required emergent intubation in

\section{QUICK LOOK}

\section{Current knowledge}

Endotracheal intubation for patients with the 2019 coronavirus (COVID-19) places clinical staff at elevated risk of contracting COVID-19. An intubation checklist can reduce risk and thus acts as a potential safeguard. Specific, step-by-step guidance to perform safe endotracheal intubation in the setting of COVID-19 are limited.

\section{What this paper contributes to our knowledge}

This article outlines the development and refinement of a COVID-19 intubation checklist and operational protocol for ICU staff. The checklist serves as a pragmatic bedside tool for clinicians, and the described process of checklist development may serve as a model for facilities preparing pandemic protocols.

the ICU. This initial run-through included an intubating physician, respiratory therapist, and 2 nurses (ie, a code nurse at bedside and a Virtual Critical Care nurse working remotely, but who was present via 2-way video to document medication administration). After intubation was completed successfully, an immediate debrief identified a number of previously unanticipated issues, including challenging communication between participating staff, delayed medication procurement, and minor yet critical errors in equipment setup specific to the new COVID intubation protocol. These deficiencies were summarized, circulated via email among the ICU staff, and discussed, allowing for further refinement of the intubation checklist.

All relevant members of the multidisciplinary ICU team were included in this process to refine the checklist. The mock COVID intubation exercise exposed issues with the rapid-sequence intubation (RSI) kits (to be used for all intubations of patients with suspected or confirmed COVID19), particularly pertaining to the neuromuscular blocking agents. Two areas for improvement were identified. First, additional neuromuscular blocking agents were added to the RSI kit. Second, the correct doses of all medications in the RSI kit were not known to everyone involved. The ICU pharmacist created simple weight-based dosing charts for all medications included and these charts were then printed and secured to each RSI kit. Furthermore, the ICU respiratory therapists were trained on appropriate assembly of the ventilator circuit, using high-efficiency particulate air filters attached to the airway and the importance of having all necessary equipment in the patient's room at the outset.

The facility's ICU nurse leadership and educator ensured that dedicated training was available for day, night, and weekend staff. Multiple mock sessions were scheduled over several weeks to maximize reach to all ICU staff. 
Table 1. Results of Measure Variables for First Run Versus Second Run

\begin{tabular}{|c|c|c|c|c|c|c|c|c|c|}
\hline & \multirow{3}{*}{ Participants, $n$} & \multirow{2}{*}{\multicolumn{2}{|c|}{ Time to Completion }} & \multirow{2}{*}{\multicolumn{2}{|c|}{ Checklist Adherence, $\%$}} & \multicolumn{4}{|c|}{ Communication Measures } \\
\hline & & & & & & \multicolumn{2}{|c|}{ Roles Assigned } & \multicolumn{2}{|c|}{ Closed-Loop Communication } \\
\hline & & First & Second & First & Second & First & Second & First & Second \\
\hline Simulation \#1 & 4 & 9:05 & $5: 28$ & 72.2 & 100 & No & Yes & No & Yes \\
\hline Simulation \#2 & 4 & 10:07 & $3: 26$ & 61.1 & 100 & Yes & Yes & No & Yes \\
\hline Simulation \#3 & 5 & 7:05 & $4: 02$ & 88.9 & 100 & No & Yes & No & Yes \\
\hline Simulation \#4 & 4 & $9: 29$ & $3: 24$ & 77.8 & 100 & Yes & Yes & No & Yes \\
\hline Average, time or $\%$ & NA & $8: 57$ & $4: 08$ & 75.0 & 100 & 50 & 100 & 0 & 100 \\
\hline
\end{tabular}

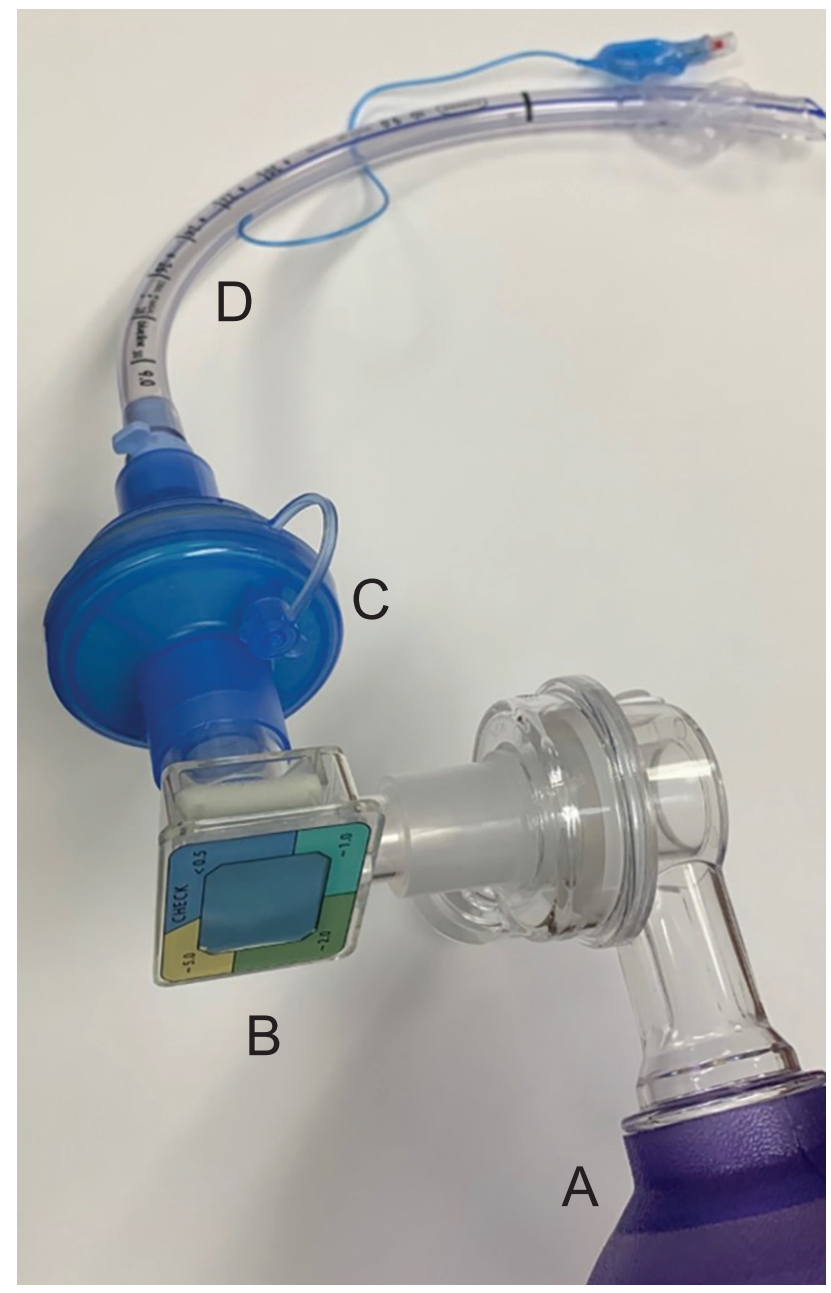

Fig. 1. Suggested endotracheal tube set-up with $(A)$ manual resuscitation bag, (B) capnometer, (C) viral filter, and (D) endotracheal tube.

Each session involved 2 back-to-back practice runs, the first to familiarize staff with the new checklist and to identify errors, the second to develop comfort and proficiency. Between each practice run, individualized feedback was provided to each participant. One session was recorded to enable asynchronous learning for clinicians and nurses unable to attend live sessions.

\section{Results}

For 4 of these sessions, data were collected on simulation completion time, checklist adherence, and specific communication measures (eg, assignment of roles and use of closed-loop communication during the simulation). A total of 10 nurses, 2 advanced practice providers, and 5 respiratory therapists participated in these 4 simulations. Table 1 summarizes the results. Compared to the first run-through, the second run-through required less time to complete (average 8:57 vs 4:08), and witnessed increased adherence to all checklist items (75\% vs $100 \%)$. The use of important communication measures, such as role assignment $(50 \%$ vs $100 \%$ ) and closed loop communication (0\% vs $100 \%)$, also improved. One-way analysis of variance calculation for each category was nonsignificant.

Our facility's COVID-19-specific advanced airway is shown in Figure 1, and the recommended ancillary airway equipment is highlighted in Figure 2. While not specifically part of the protocol, the use of an intubation tent, box, or something similar, if available, can be considered to minimize staff exposure to aerosolized secretions (Fig. 3). The finalized intubation preparation sheet and checklist can be found in the supplementary materials (available at http:// www.rcjournal.com).

\section{Discussion}

The described intubation checklist provides a practical bedside tool for suspected or confirmed COVID-19 patients with acute respiratory failure who require invasive mechanical ventilation. Analysis suggests that use of the protocol can reduce the time for intubation and improve communication between staff. Equally as important as the checklist itself is the multidisciplinary and iterative process 


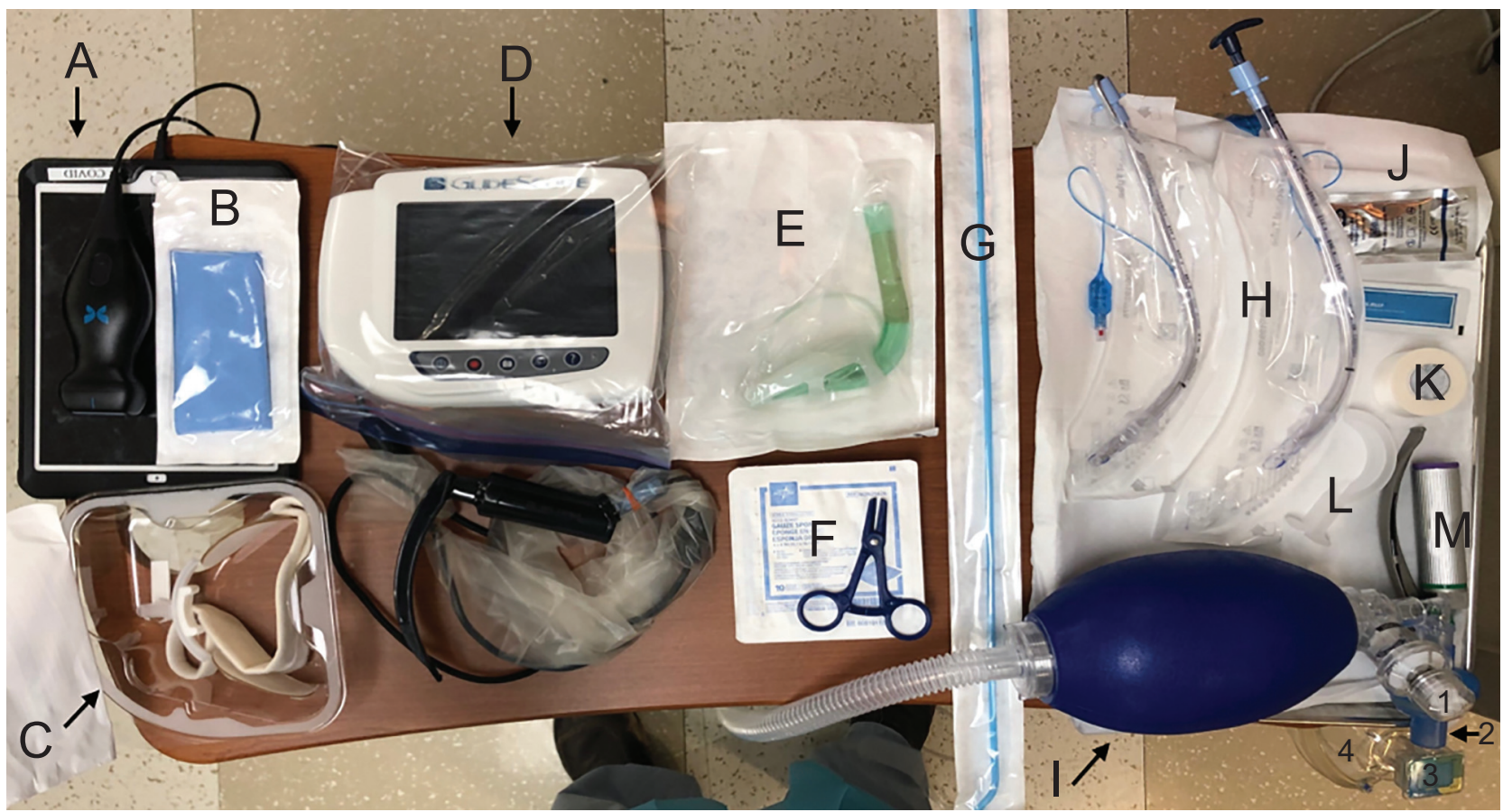

Fig. 2. Ancillary equipment prepared in advance for transport into the patient's room prior to endotracheal intubation. A: Point of care ultrasound; (B) ultrasound probe cover; (C) tube holder and strap; (D) video laryngoscopy screen and blade with ultrasound probe cover over wire; (E) laryngeal mask airway; (F) disposable clamp and gauze; (G) bougie introducer; $(\mathrm{H})$ primary and back-up endotracheal tubes, preloaded with stylet(s); (I) manual resuscitation bag preloaded with PEEP valve, high-efficiency particulate air viral filter, capnometer, and mask; (J) lubricant (sterile and nonsterile); (K) silk tape; (L) oropharyngeal airway; and (M) direct laryngoscopy light and blade.

used in its development. All relevant stakeholders-physicians, advanced practice providers, nurses, respiratory therapists, and pharmacists - were empowered to provide feedback. Dedicated training sessions spread out across multiple shifts not only provided crucial practice for the ICU staff but also allowed for the identification of critical gaps related to communication, process, and equipment. Identification of these gaps before the ICU's first intubation of a patient with COVID-19 was crucial to optimize patient and staff safety. This approach mirrors the recommended timeline of activities in caring for patients infected with high-containment pathogens: staging equipment, implementing staffing plans, conducting team meetings, debriefing stakeholders, and refining clinical protocols. ${ }^{15}$

The primary goal of the COVID-19 intubation checklist is to optimize safety of the ICU staff during a highrisk situation by minimizing aerosolization of secretions, the number of staff required in the room, the time spent in the room, and the frequency of donning/doffing PPE. Under normal circumstances for intubation, the ICU door remains open and a "runner" nurse and ICU pharmacist are present for unanticipated equipment or medication needs. Back-up equipment, such as a bougie introducer, is available in a prestocked airway cart situated outside the patient's room. No viral filter is used, Virtual Critical Care is not normally involved, and a second airway operator is not typically present. Moreover, choice of intubation mode (direct vs video laryngoscopy) and sedation medications are the purview of the intubating clinician. Under COVID-19 circumstances, such flexibilities could become liabilities that increase the risk of delays and accidental contamination and exposure. Thus, in developing the COVID-19 checklist, every effort was made to streamline the sequence of events to ensure uniformity between diverse operators using a minimal number of additional staff. The checklist's use facilitates communication between team members during a high-stress procedure where even small errors could have serious repercussions.

Our proposed COVID-19 checklist is not without limitations. First, currently available evidence remains unclear as to whether intubation checklists improve patient outcomes in a meaningful way. Second, different centers will have different equipment, processes of care, and resource availability. Thus, this checklist is not a "one size fits all" solution; it will need further adaptation for individual facilities and might not be feasible if supply-chain disruptions lead to equipment shortages. Third, use of the checklist may not be feasible in truly emergent situations, such as self-extubation in a patient with COVID-19 requiring immediate re-intubation. Fourth, the checklist must be revisited and refined continuously as recommendations surrounding COVID-19 best practices evolve. Lastly, the checklist is not externally validated. A future validation study using rigorous scientific methods will 


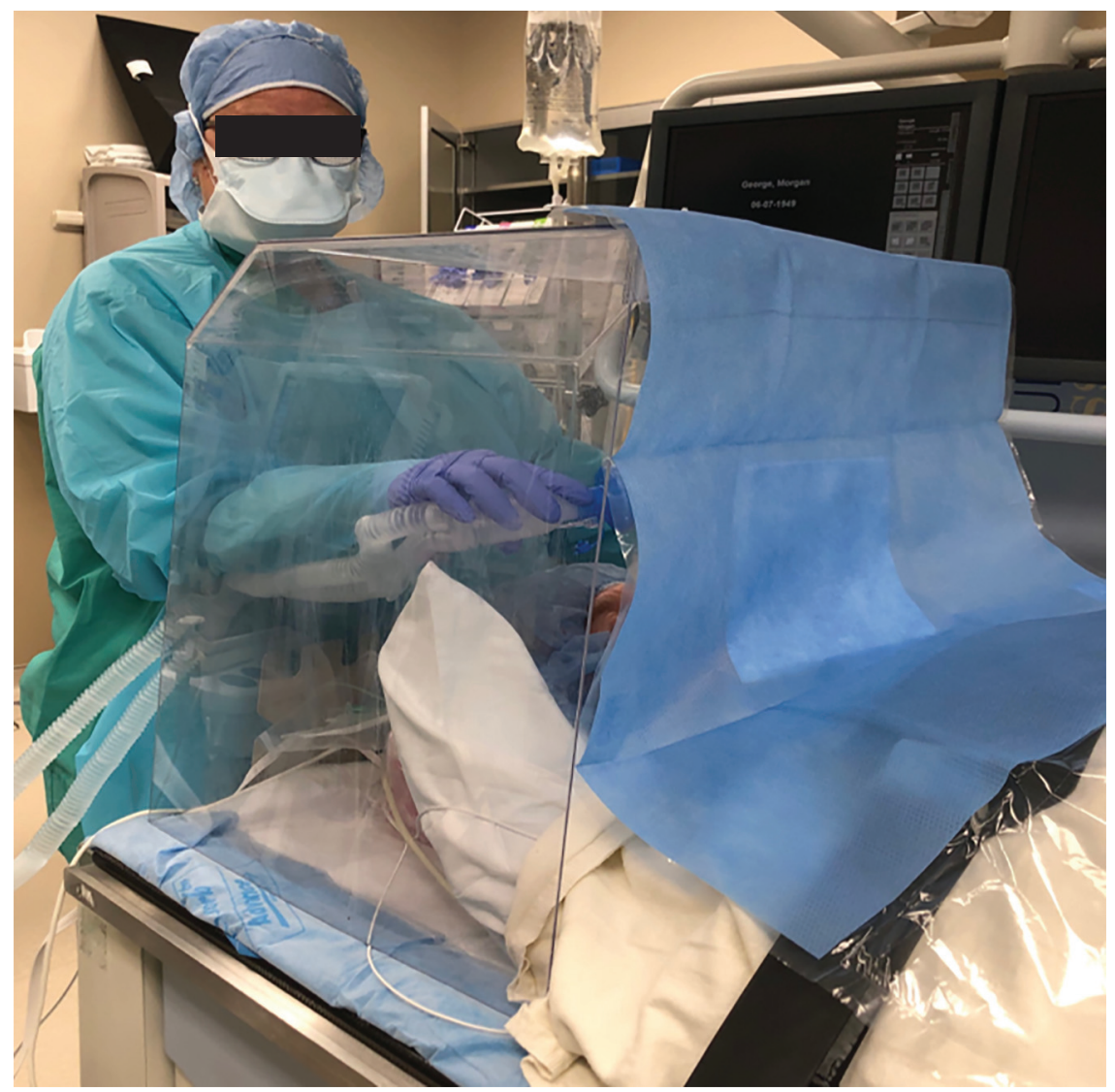

Fig. 3. Optional intubation box.

be important. Given the climate of urgency created by the COVID-19 pandemic, there was insufficient time to perform a true validation study prior to clinical implementation of this protocol at our facility. Because the imperative to "do something" was great, ${ }^{16}$ we opted to adapt a familiar institutional protocol using many common-sense features. Our experience to date suggests widespread acceptance and practical utility of the protocol in our ICU.

\section{Conclusions}

We present an endotracheal intubation checklist, which serves as a pragmatic bedside tool when intubating patients with COVID-19 who are experiencing advanced respiratory failure. Using multidisciplinary discussion and dedicated practice sessions, this checklist was refined to optimize safety and communication during a high-risk procedure. The checklist can be adapted to fit the resources and culture of each facility that uses it.

\section{ACKNOWLEDGMENTS}

The authors wish to thank the Atrium Health Respiratory Therapy department for its invaluable contributions to the clinical management of COVID-19 patients throughout the system.

\section{REFERENCES}

1. Ng K, Poon BH, Kiat Puar TH, Shan Quah JL, Loh WJ, Wong YJ, et al. COVID-19 and the risk to health care workers: a case report. Ann Intern Med 2020;172(11):766-767.

2. Mira JJ, Asencio-Aznar A, Gea T, Lorenzo S, Antón P. Measures to assure that ebola guidelines are correctly applied. Med Princ Pract 2016;25(2):191-195.

3. Kwon JH, Burnham CD, Reske KA, Liang SY, Hink T, Wallace MA, et al. Assessment of healthcare worker protocol deviations and self-contamination during personal protective equipment donning and doffing. Infect Control Hosp Epidemiol 2017;38(9):10771083.

4. Haynes AB, Weiser TG, Berry WR, Lipsitz SR, Breizat AH, Dellinger EP, et al. A surgical safety checklist to reduce morbidity and mortality in a global population. N Engl J Med 2009;360(5):491-499.

5. Sevilla-Berrios R, O'Horo JC, Schmickl CN, Erdogan A, Chen X, Garcia Arguello LY, et al. Prompting with electronic checklist improves clinician performance in medical emergencies: a high-fidelity simulation study. Int J Emerg Med 2018;11(1):26.

6. Urayeneza O, Mujyarugamba $P$, Rukemba $Z$, Nyiringabo V, Ntihinyurwa $P$, Baelani JI, et al. Increasing evidence-based interventions in patients with acute infections in a resource-limited setting: a before-and-after feasibility trial in Gitwe, Rwanda. Intensive Care Med 2018;44(9):1436-1446.

7. Wichmann D, Belmar Campos CE, Ehrhardt S, Kock T, Weber C, Rohde $\mathrm{H}$, et al. Efficacy of introducing a checklist to reduce central venous line associated bloodstream infections in the ICU caring for adult patients. BMC Infect Dis 2018;18(1):267. 


\section{INTUBATION CHECKLIST FOR COVID-19}

8. Janz DR, Semler MW, Joffe AM, Casey JD, Lentz RJ, deBoisblanc $\mathrm{BP}$, et al. A multicenter randomized trial of a checklist for endotracheal intubation of critically ill adults. Chest 2018;153(4):816-824.

9. Klingberg C, Kornhall D, Gryth D, Krüger AJ, Lossius HM, Gellerfors M. Checklists in pre-hospital advanced airway management. Acta Anaesthesiol Scand 2020;64(1):124-130.

10. Zuo M, Huang Y, Ma W, Xue Z, Zhang J, Gong Y, Che L, Gong Y-L, et al. Expert recommendations for tracheal intubation in critically ill patients with noval coronavirus disease 2019. Chin Med Sci J 2020 [Epub ahead of print] doi: 10.24920/003724.

11. Trujillo A. Response to Wen and Li. Anesthesia procedure of emergency operation for patients with suspected or confirmed COVID-19. Surg Infect (Larchmt) 2020;21(4):398.
12. Missair A, Marino MJ, Vu CN, Gutierrez J, Missair A, Osman B, Gebhard RE. Anesthetic implications of ebola patient management: a review of the literature and policies. Anesth Analg 2015;121(3):810821.

13. Chavez S, Long B, Koyfman A, Liang SY. Coronavirus disease (COVID-19): a primer for emergency physicians. Am J Emerg Med 2020 [Epub ahead of print] doi: 10.1016/j.ajem.2020.03.036.

14. Kamming D, Gardam M, Chung F. Anaesthesia and SARS. Br J Anaesth 2003;90(6):715-718.

15. Garibaldi BT, Chertow DS. High-containment pathogen preparation in the intensive care unit. Infect Dis Clin North Am 2017;31(3):561-576.

16. Angus DC. Optimizing the trade-off between learning and doing in a pandemic. JAMA 2020;323(19):1895-1896. 